\title{
Acute sinusitis complicated with Pott puffy tumour
}

\author{
Petros Koltsidopoulos MD PhD, Eleni Papageorgiou MD PhD, Charalampos Skoulakis MD PhD
}

Cite as: CMAJ 2019 February 11;191:E165. doi: 10.1503/cmaj.181025

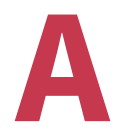

37-year-old woman presented to the department of otolaryngology with a 15-day history of frontal headache. The patient had been treated for sinusitis with a 10-day course of amoxicillin and clavulanic acid without any improvement. Five days before presentation, a well-defined, fluctuating forehead swelling had developed (Figure 1). The patient was afebrile and fully alert and oriented. Nasal endoscopy showed bilateral mucopurulent discharge. The neurologic examination was unremarkable. Magnetic resonance imaging (MRI) showed bilateral opacification of all paranasal sinuses, osteomyelitis of the frontal bone with erosion of anterior frontal sinus wall and the presence of a subperiosteal abscess in the patient's forehead (Figure 2). We diagnosed acute sinusitis complicated with Pott puffy tumour. We took a combined endoscopic and external surgical approach, with drainage of the abscess and débridement of necrotic material. Cultures grew Staphylococcus aureus. We treated the patient with antibiotics for 6 weeks after surgery.

First described in 1768 by Sir Percivall Pott, Pott puffy tumour is a subperiosteal abscess of the anterior wall of the frontal sinus, associated with underlying frontal osteomyelitis. ${ }^{1}$ It is usually related to frontal sinusitis. However, it may also occur as a result of head trauma, surgery in the frontal region, cocaine abuse or dental infections. Pott puffy tumour can be found in patients of all ages, with a higher incidence among adolescents. ${ }^{2}$ It is an uncommon and potentially dangerous complication as it can be accompanied by intracranial pathology, such as meningitis, epidural and brain abscess. The infection can spread directly by erosion of the posterior wall of frontal sinus or by migration of septic thrombi through the diploic veins to the dura. ${ }^{1}$

Early diagnosis and immediate management are essential to avoid intracranial complications. Diagnosis of Pott puffy tumour is based on clinical and radiological findings. Computed tomography scan is considered the imaging modality of choice to confirm the diagnosis. However, MRI is better for detecting intracranial involvement. ${ }^{3}$

Treatment of Pott puffy tumour consists of surgical drainage of the abscess, removal of necrotic material and a 6- to 8-week antibiotic course. ${ }^{2,4}$ Craniotomy might be required if it is associated with an intracranial lesion. ${ }^{3}$

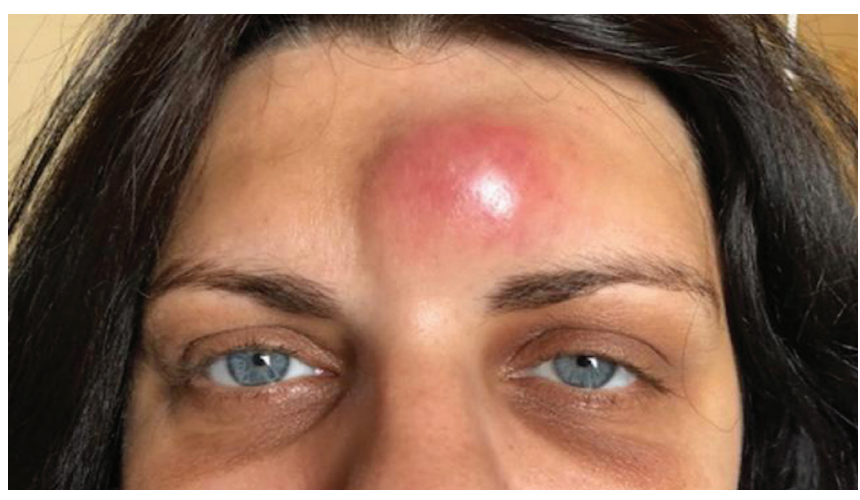

Figure 1: Clinical photograph showing a soft, well-demarcated swelling of the forehead of a 37-year-old woman.
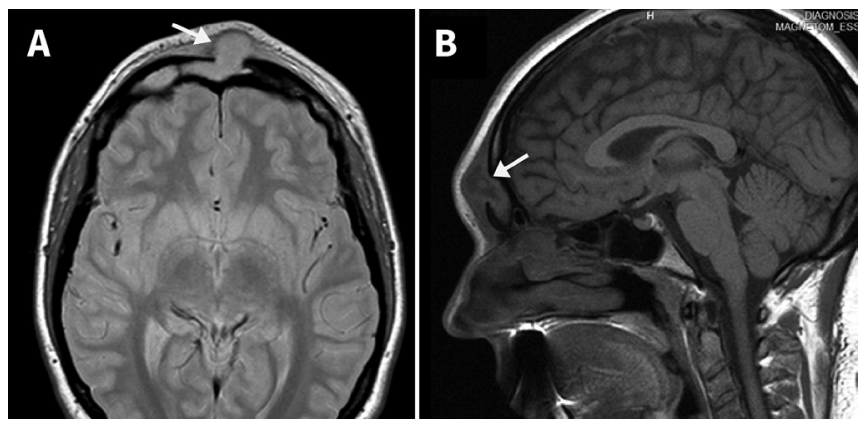

Figure 2: (A) Axial $T_{2}$-weighted magnetic resonance image (MRI) showing a subperiosteal abscess (arrow) in the forehead of a 37-year-old woman. (B) Sagittal $T_{1}$-weighted MRI of paranasal sinuses showing osteomyelitis of the frontal bone with erosion of anterior frontal sinus wall (arrow).

\section{References}

1. Goldberg AN, Oroszlan G, Anderson TD. Complications of frontal sinusitis and their management. Otolaryngol Clin North Am 2001;34:211-25.

2. Forgie SE, Marrie TJ. Pott's puffy tumor. Am J Med 2008;121:1041-2.

3. Kombogiorgas D, Solanki GA. The Pott puffy tumor revisited: neurosurgical implications of this unforgotten entity. Case report and review of the literature. J Neurosurg 2006;105(Suppl2):143-9.

4. Akiyama K, Karaki M, Mori N. Evaluation of adult Pott's puffy tumor: our five cases and 27 literature cases. Laryngoscope 2012;122:2382-8.
Competing interests: None declared.

This article has been peer reviewed.

The authors have obtained patient consent.
Affiliations: Department of Otolaryngology (Koltsidopoulos), General Hospital of Volos, Volos, Greece; Departments of Ophthalmology (Papageorgiou) and Otolaryngology (Skoulakis), University Hospital of Larissa, Larissa, Greece.
Correspondence to: Petros Koltsidopoulos, petkoltsid@yahoo.gr 